\title{
Relationship of ERCC5 genetic polymorphisms with metastasis and recurrence of gastric cancer
}

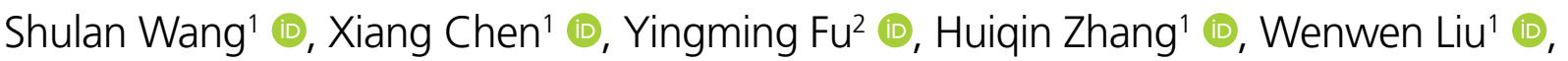

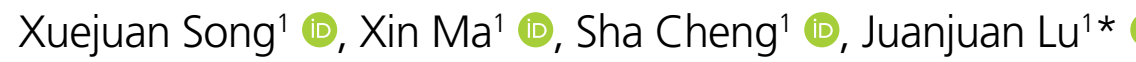

\section{SUMMARY}

OBJECTIVE: This study aims to explore the role of ERCC5 genetic polymorphisms in gastric cancer and their relationship with metastasis and recurrence of gastric cancer.

METHODS: A total of 200 patients with gastric cancer and 133 healthy subjects were enrolled. MassARRAY iPLEX ${ }^{\circledR}$ technology was used to genotype ERCC5 rs2016073, rs751402, rs2094258, rs2296147, and rs2296148 between the control group and the gastric cancer group. The relationship of ERCC5 genetic polymorphisms with metastasis and recurrence of gastric cancer was explored. The differences in sociodemographic characteristics between patients with gastric cancer and control subjects were compared using the chi-square test. The genetic loci between the control group and the gastric cancer group were analyzed by the chi-square test.

RESULTS: There was no significant difference in the metastasis of gastric cancer between males and females $(p=0.628)$, but there was a significant difference in the metastasis of gastric cancer $(p=0.005)$. Patients aged $\leq 60$ years and $>60$ years showed no significant difference in the metastasis of gastric cancer $(p=0.420)$, but there was a significant difference in the recurrence of gastric cancer $(p<0.001)$. The loci rs2016073, rs751402, and rs2094258 in the gastric cancer group showed no significant differences compared with the control group ( $p=0.194)$, and the loci rs2296147 and rs2296148 showed significant differences.

CONCLUSIONS: The results suggested that ERCC5 polymorphisms (e.g., rs201607, rs751402, rs2094258, rs2296147, and rs2296148) may be associated with metastasis and recurrence of gastric cancer.

KEYWORDS: ERCC5. Genetic polymorphism. Metastasis. Recurrence. Gastric cancer.

\section{INTRODUCTION}

Gastric cancer is the second most common cause of cancer-related deaths in the world, the epidemiology of which has changed within the past decades ${ }^{1}$. Gastric cancer remains a considerable health burden throughout the world ${ }^{2}$. Metastasis is a crucial impediment to the successful treatment of gastric cancer $^{3}$. Recurrence of gastric cancer after 10 years of surgical resection is highly rare, and local recurrence is common after radical surgery ${ }^{4}$. Many studies have explored biomarkers to monitor metastasis and recurrence of gastric cancer ${ }^{5-7}$, but further study is still needed.

The common polymorphic variant in the 5'-untranslated region of the excision repair cross-complementation group 5 (ERCC5) gene was described to generate an upstream open reading frame that regulates both the basal ERCC5 expression and its ability to be synthesized following DNA damage ${ }^{8}$. Genetic variations in ERCC5 might influence individual vulnerability to gastric cancer ${ }^{9}$.

\footnotetext{
'Gansu Provincial Hospital, Department of Digestive - Lanzhou (Gansu) China.

${ }^{2}$ Wushan County Hospital, Department of Digestive - Tianshui (Gansu), China.

*Corresponding author: jj_lu19@163.com

Conflicts of interest: the authors declare there is no conflicts of interest. Funding: the study was supported by the Scientific Research Plan Project of Health Industry of Gansu Province (GSWSKY2018-22).

Received on August 04, 2021. Accepted on September 07, 2021.
} 
A previous study indicated that ERCC5 rs2094258 polymorphism may contribute to the risk of breast cancer ${ }^{10}$. Our previous study found that ERCC5 rs2094258 and rs751402 polymorphisms were not associated with the development of gastric cancer in co-dominant, dominant, and recessive models ${ }^{11}$. But in a meta-analysis, it was demonstrated that rs751402 C>T, rs2296147 T>C, and rs873601 G>A polymorphisms of ERCC5 gene were associated with the susceptibility of gastric cancer ${ }^{12}$. In another study, the ERCC5 rs751402 gene polymorphism may influence the susceptibility to gastric cancer in the Chinese population ${ }^{13}$.

In this study, the roles of ERCC5 genes (e.g., rs2016073, rs751402, rs2094258, rs2296147, and rs2296148) in gastric cancer were explored, and their relationship with metastasis and recurrence of gastric cancer was studied by MassARRAY iPLEX $^{\circledast}$ technology.

\section{METHODS}

\section{Study population}

In this study, 333 subjects were enrolled from our hospital between October 2012 and December 2014, including 200 patients with gastric cancer who were newly diagnosed as case group and 133 subjects as control group. The case group included 170 men and 30 women, with age ranging from 39 to 78 years $(56.9 \pm 10.1)$. The control group included 80 men and 53 women, with age ranging from 20 to 87 years $(49.4 \pm 14.2)$. The percentage of metastasis and recurrence in the case group was $9.0 \%$ and $18.0 \%$, respectively. Exclusion criteria for patients with gastric cancer were primary tumors outside the esophagus, other malignant neoplasms, recurrent tumors, and serious liver and kidney diseases. Exclusion criteria for the control group were a history of malignant cancers, serious liver and kidney diseases, and digestive system disease. The basic medical data of patients with gastric cancer were obtained from medical records. A standardized questionnaire including sociodemographic characteristics was used to interview both patients and controls. Before the study, all subjects signed the informed consent, and this study was approved by our hospital.

\section{DNA extraction and single-nucleotide polymorphisms genotyping}

DNA from peripheral blood samples that were collected from patients was extracted using TIANamp Blood DNA Kit (Tiangen, Beijing, China), according to the instructions of the manufacturer. The ERCC5 rs2016073, rs751402, rs2094258, rs2296147, and rs2296148 were genotyped by MassARRAY iPLEX ${ }^{\circledR}$ technology (Shanghai Genechem Co., Ltd.). The polymerase chain reaction (PCR) fragments of the investigated polymorphisms were subsequently digested with their specific restriction enzyme. The PCR began with an initial denaturation at $95^{\circ} \mathrm{C}$ for $2 \mathrm{~min}$, followed by 45 cycles of denaturation at $95^{\circ} \mathrm{C}$ for $30 \mathrm{~s}$, annealing at $56^{\circ} \mathrm{C}$ for $30 \mathrm{~s}$, extension at $72^{\circ} \mathrm{C}$ for $60 \mathrm{~s}$, and a final extension at $72^{\circ} \mathrm{C}$ for $5 \mathrm{~min}$. After desalted with resin, the Typer software automatically interpreted the molecular weight peaks detected by the mass spectrometry, and the transformation showed the molecular mass spectrum peak map corresponding to the single-nucleotide polymorphism (SNP) site. The PCR primers were as follows:

$\begin{array}{llr}\text { rs2016073 } & \text { ACGTTGGATGCTCCTITGGAAAGGCTTATC } & \text { (2nd-PCRP) } \\ & \text { ACGTTGATGAAGCAGGAAGGGCTCTAGG } & \text { (1st-PCRP) } \\ \text { rs751402 } & \text { ACGTTGGATGGTATAGACGGAACCGAGC } & \text { (2nd-PCRP) } \\ & \text { ACGTTGGATGAACAGCCAGAAGATGTCCC } & \text { (1st-PCRP) } \\ \text { rs2094258 } & \text { ACGTTGGATGCAATTCCCGTATACTCTG } & \text { (2nd-PCRP) } \\ & \text { ACGTGGATGAACTCAGTGAAAAGGCTGAC } & \text { (1st-PCRP) } \\ \text { rs2296147 } & \text { ACGTGGATGCAGACGTTGGGCCTAAGC } & \text { (2nd-PCRP) } \\ & \text { ACGTGGATGAACACGTCTCAGCAGCTGTC } & \text { (1st-PCRP) } \\ \text { rs2296148 } & \text { ACGTTGGATGATTCTTCTACGACGGACTGC } & \text { (2nd-PCRP) } \\ & \text { ACGTTGGATGCTTTGTTGTGTAGGAGCAGG } & \text { (1st-PCRP) }\end{array}$

\section{Statistical analysis}

Statistical analyses were performed using SPSS version 17.0 software (SPSS Inc., Chicago, IL, USA). The differences in sociodemographic characteristics between patients with metastasis and recurrence of gastric cancer were compared using the chi-square test. Genetic loci between the control group and the gastric cancer group were analyzed using the chi-square test. All tests were two-sided. A p-value $<0.05$ was considered statistically significant.

\section{Ethical issue and informed consent}

The authors declare that they have no conflict of interest. All procedures performed in studies involving human participants were in accordance with the ethical standards of the Institutional and/or National Research Committee and with the 1964 Helsinki Declaration and its later amendments or comparable ethical standards. This article does not contain any studies with animals performed by any of the authors. Informed consent was obtained from all individual participants included in the study. The study protocol and the comprehensive written informed consent used in this study protocol were reviewed. 


\section{RESULTS}

\section{Relationship of gender and age with} metastasis and recurrence of gastric cancer In patients with metastasis, there were 16 males and 2 females. In patients without metastasis, there were 154 males and 28 females. There was no significant difference in the metastasis of gastric cancer between males and females $(\mathrm{p}=0.628)$. In patients with recurrence, there were 36 males and 0 female. In patients without recurrence, there were 134 males and 30 females. Results showed that the recurrence rate of males was higher than that of females $(\mathrm{p}=0.005)$. Among patients aged $\leq 60$ years, patients with metastasis were 13 , and patients without metastasis were 114. Among patients aged $>60$ years, patients with metastasis were 5 , and patients without metastasis were 68 . There was no significant difference in age for the metastasis of gastric cancer. Among patients aged $\leq 60$ years, patients with recurrence were 0 , and patients without recurrence were 126. Among patients $>60$ years, patients with recurrence were 36 , and patients without recurrence were 60 . There was a significant difference in age for the recurrence of gastric cancer $(\mathrm{p}<0.001)$ (Table 1$)$.

\section{Comparison of genetic loci between the control group and the gastric cancer group} As for loci rs2016073, rs751402, and rs2094258, there were no significant differences between the control group and the gastric cancer group $(\mathrm{p}=0.194, \mathrm{p}=0.257$, and $\mathrm{p}=0.686$, respectively). As for loci rs2296147 and rs2296148, there were significant differences between the control group and the gastric cancer group ( $\mathrm{p}<0.001$ and $\mathrm{p}=0.005$, respectively) (Table 2 ).

\section{Relationship of gene loci with metastasis and recurrence of gastric cancer}

As for locus rs2016073, there was a significant difference between different genotypes and gastric cancer metastasis

Table 1. Relationship of gender and age with metastasis and recurrence of gastric cancer.

\begin{tabular}{|c|c|c|c|c|c|c|}
\hline & \multicolumn{3}{|c|}{ Metastasis } & \multicolumn{3}{|c|}{ Recurrence } \\
\hline & Yes & No & $\mathrm{p}$-value & Yes & No & $p$-value \\
\hline \multicolumn{7}{|l|}{ Gender } \\
\hline Male & 16 & 154 & \multirow{2}{*}{0.628} & 36 & 134 & \multirow{2}{*}{0.005} \\
\hline Female & 2 & 28 & & 0 & 30 & \\
\hline \multicolumn{7}{|l|}{ Age, year } \\
\hline$\leq 60$ & 13 & 114 & \multirow{2}{*}{0.420} & 0 & 126 & \multirow{2}{*}{$<0.001$} \\
\hline$>60$ & 5 & 68 & & 36 & 60 & \\
\hline
\end{tabular}

$(\mathrm{p}=0.021)$. As for locus rs751402, there was a significant difference between different genotypes and gastric cancer metastasis $(\mathrm{p}=0.021)$. As for locus rs2094258, there was a significant difference between different genotypes and gastric cancer metastasis $(\mathrm{p}<0.001)$. As for locus rs 2296147 , there was a significant difference between different genotypes and gastric cancer metastasis $(\mathrm{p}<0.001)$. There was no significant difference between different genotypes of rs2296148 locus and gastric cancer metastasis $(\mathrm{p}=0.197)$ (Table 3).

As for locus rs2016073, there was no significant difference between different genotypes and gastric cancer recurrence. As for locus rs751402, there was no significant difference between different genotypes and gastric cancer recurrence $(\mathrm{p}=0.094)$. As for locus rs2094258, there was a significant difference between different genotypes and gastric cancer recurrence $(\mathrm{p}=0.021)$. As for locus rs2296147, there was no significant difference between different genotypes and gastric cancer recurrence $(\mathrm{p}=0.434)$. As for locus rs2296148, there was a significant difference between different genotypes and gastric cancer recurrence $(\mathrm{p}<0.001)$ (Table 3$)$.

\section{DISCUSSION}

Functions pertaining to DNA repair and synthesis are believed to play a critical role in cancer development and seem to be affected by genetic polymorphisms ${ }^{14}$. Several SNPs in ERCC5 gene have been identified and have been studied for their association with different cancer risk, such as rs751402 C>T, rs2296147 T>C, rs873601 G>A, rs2094258 C>T, rs 1047768 $\mathrm{T}>\mathrm{C}, \mathrm{rs} 17655 \mathrm{G}>\mathrm{C}, \mathrm{rs} 2018836 \mathrm{G}>\mathrm{A}$, and $\mathrm{rs} 3818356 \mathrm{G}>\mathrm{A}^{12,15-17}$. In this study, a case-control study was performed to investigate the association of ERCC5 genetic polymorphisms with metastasis and recurrence of gastric cancer.

In a previous study, ERCC5-763 A>G (rs2016073) may be associated with chemosensitivity of oxaliplatin-based chemotherapy in Chinese patients with advanced colorectal cancer ${ }^{18}$. In our study, locus rs2016073 showed no significant differences between the control group and the gastric cancer group and between different genotypes and gastric cancer recurrence, but a significant difference between different genotypes and gastric cancer metastasis was observed. So, results indicated that locus rs2016073 may play a role in gastric cancer, especially in metastasis.

Our results found that locus rs751402 in the gastric cancer group showed no significant difference in the control group and in the gastric cancer recurrence group, which was consistent with our previous study ${ }^{11}$. Another study using unconditional multiple logistic regression analysis revealed that the AA genotype of rs751402 significantly increased risk of gastric cancer compared with the GG genotype ${ }^{16}$. Stratification by cancer type 
Table 2. Comparison of genetic loci between the control group and the gastric cancer group.

\begin{tabular}{|c|c|c|c|c|c|}
\hline Loci & $\begin{array}{l}\text { Single-nucleotide } \\
\text { polymorphisms }\end{array}$ & Control group & Gastric cancer group & $\chi^{2}$ & $p$-value \\
\hline \multirow{3}{*}{ rs2016073 } & A & 67 & 90 & \multirow{3}{*}{3.281} & \multirow{3}{*}{0.194} \\
\hline & G & 20 & 22 & & \\
\hline & $A G$ & 46 & 88 & & \\
\hline \multirow{3}{*}{ rs751402 } & A & 20 & 22 & \multirow{3}{*}{2.72} & \multirow{3}{*}{0.257} \\
\hline & G & 67 & 90 & & \\
\hline & GA & 48 & 88 & & \\
\hline \multirow{3}{*}{ rs2094258 } & $C$ & 47 & 44 & \multirow{3}{*}{0.753} & \multirow{3}{*}{0.686} \\
\hline & $\mathrm{T}$ & 16 & 21 & & \\
\hline & CT & 72 & 76 & & \\
\hline \multirow{3}{*}{ rs2296147 } & $C$ & 4 & 7 & \multirow{3}{*}{17.11} & \multirow{3}{*}{$<0.001$} \\
\hline & $T$ & 76 & 153 & & \\
\hline & CT & 55 & 40 & & \\
\hline \multirow{2}{*}{ rs2296148 } & C & 128 & 170 & \multirow{2}{*}{7.902} & \multirow{2}{*}{0.005} \\
\hline & TC & 7 & 30 & & \\
\hline
\end{tabular}

Table 3. Relationship of gene loci with metastasis and recurrence of gastric cancer.

\begin{tabular}{|c|c|c|c|c|c|c|c|c|c|}
\hline \multirow{2}{*}{ Loci } & \multirow{2}{*}{$\begin{array}{l}\text { Single-nucleotide } \\
\text { polymorphisms }\end{array}$} & \multicolumn{2}{|c|}{ Metastasis } & \multirow{2}{*}{$\chi^{2}$} & \multirow{2}{*}{ p-value } & \multicolumn{2}{|c|}{ Recurrence } & \multirow{2}{*}{$\chi^{2}$} & \multirow{2}{*}{ p-value } \\
\hline & & Yes & No & & & Yes & No & & \\
\hline \multirow{3}{*}{ rs2016073 } & G & 22 & 0 & \multirow{3}{*}{7.758} & \multirow{3}{*}{0.021} & 1 & 21 & \multirow{3}{*}{4.721} & \multirow{3}{*}{0.094} \\
\hline & A & 89 & 1 & & & 15 & 75 & & \\
\hline & GA & 80 & 8 & & & 21 & 67 & & \\
\hline \multirow{3}{*}{ rs751402 } & $A G$ & 80 & 8 & \multirow{3}{*}{7.758} & \multirow{3}{*}{0.021} & 21 & 67 & \multirow{3}{*}{4.721} & \multirow{3}{*}{0.094} \\
\hline & $\mathrm{G}$ & 89 & 1 & & & 15 & 75 & & \\
\hline & $A$ & 22 & 0 & & & 1 & 21 & & \\
\hline \multirow{3}{*}{ rs2094258 } & $C$ & 52 & 9 & \multirow{3}{*}{21.475} & \multirow{3}{*}{$<0.001$} & 9 & 52 & \multirow{3}{*}{7.758} & \multirow{3}{*}{0.021} \\
\hline & $\mathrm{CT}$ & 109 & 0 & & & 28 & 81 & & \\
\hline & $\mathrm{T}$ & 30 & 0 & & & 0 & 30 & & \\
\hline \multirow{3}{*}{ rs2296147 } & $C$ & 6 & 1 & \multirow{3}{*}{31.131} & \multirow{3}{*}{$<0.001$} & 0 & 7 & \multirow{3}{*}{1.67} & \multirow{3}{*}{0.434} \\
\hline & $\mathrm{CT}$ & 32 & 8 & & & 8 & 32 & & \\
\hline & $T$ & 153 & 0 & & & 29 & 124 & & \\
\hline \multirow{2}{*}{ rs2296148 } & $C$ & 161 & 9 & \multirow{2}{*}{1.663} & \multirow{2}{*}{0.197} & 22 & 148 & \multirow{2}{*}{23.227} & \multirow{2}{*}{$<0.001$} \\
\hline & TC & 30 & 0 & & & 15 & 15 & & \\
\hline
\end{tabular}

indicated that rs 751402 polymorphism may increase the risk of gastric cancer and hepatocellular carcinoma, which was further confirmed by a false-positive report probability analysis ${ }^{19}$. We found that locus rs 751402 was related to gastric cancer metastasis. So, all these results indicated that locus rs751402 may play a role in gastric cancer, especially in metastasis.
The locus rs2094258 as an important polymorphism plays an important role in many cancers ${ }^{15,20}$. Positive Helicobacter pylori individuals with rs2094258 TT genotypes demonstrated an increased risk of gastric cancer ${ }^{21}$. The rs 2094258 polymorphism may be associated with an increased risk of gastric cancer in Southern China ${ }^{22}$. The rs2094258 interacted 
with rs2094258 and metabolic gene GSTP1 rs1695 and formed the basis for various inter-individual susceptibilities to atrophic gastritis ${ }^{23}$. Our results indicated that locus rs2094258 was significantly related to metastasis and recurrence of gastric cancer. The above results demonstrated that locus rs2094258 may be a critical DNA repair gene for gastric cancer.

The locus rs2296147 showed a significant difference between the control group and the gastric cancer group and was significantly related to metastasis of gastric cancer, but not to the recurrence of gastric cancer. A case-control study showed that the polymorphic locus on ERCC5 rs2296147 could reduce the risk of esophageal cancer, which will help further understand the pathogenesis of esophageal cancer ${ }^{24}$. ERCC5 rs $2296147 \mathrm{C}$ variant genotypes were associated with a significantly lower esophageal squamous cell carcinoma risk $^{25}$. The rs2296147 polymorphism might alter the risk of developing gastric cancer, especially the diffuse subtype ${ }^{25}$. Therefore, the rs 2296147 polymorphism could be used as surrogate markers for gastric cancer.

The locus rs2296148 showed no significant difference between the control group and the gastric cancer group and was not significantly related to metastasis of gastric cancer, but showed a significant difference in the recurrence of gastric cancer. Until now, there is no relevant literature report about locus rs2296148 for gastric cancer. Our results indicated that locus rs 2296148 may be related to the recurrence of gastric cancer.

\section{CONCLUSIONS}

In this study, five ERCC5 gene polymorphisms were explored. The results found that two loci (rs2296147 and rs2296148) in gastric cancer were significantly different from the control group, four loci (rs201607, rs751402, rs2094258, and rs2296147) were related to metastasis of gastric cancer, and two loci (rs2094258 and rs2296148) were related to the recurrence of gastric cancer. ERCC5 gene polymorphisms may perform important functions in gastric cancer and its metastasis and recurrence. Due to the small sample size, further validation by larger population-based case-control studies is needed.

\section{ETHICS APPROVAL AND CONSENT TO PARTICIPATE}

All participants signed the informed consent, and this study was approved by the Gansu Provincial Hospital.

\section{IMPACT STATEMENT}

This study aims to explore the role of ERCC5 genetic polymorphisms in gastric cancer. Genotype ERCC5 rs2016073, rs751402, rs2094258, rs2296147, and rs2296148 were detected. The loci rs2016073, rs751402, and rs2094258 in the gastric cancer group showed no significant differences compared with the control group, and loci rs2296147 and rs2296148 showed significant differences. ERCC5 polymorphisms (e.g., rs201607, rs751402, rs2094258, rs2296147, and rs2296148) may be associated with metastasis and recurrence of gastric cancer. ERCC5 gene polymorphisms may perform important functions in gastric cancer and its metastasis and recurrence.

\section{AUTHORS" CONTRIBUTIONS}

SW: Conceptualization, Data curation, Resources, Writing-original draft, Writing-review \& editing. XC: Conceptualization, Data curation, Investigation, Resources. YF: Conceptualization, Data curation, Formal analysis, Investigation, Resources. HZ: Conceptualization, Data curation, Formal analysis, Investigation, Resources. WL: Data curation, Formal analysis, Investigation, Methodology, Resources. XS: Data curation, Formal analysis, Investigation, Methodology, Resources. XM: Data curation, Formal analysis, Investigation, Methodology, Resources. SC: Data curation, Formal analysis, Investigation, Methodology, Resources. JL: Conceptualization, Data curation, Formal analysis, Investigation, Project administration, Resources, Supervision, Writing -original draft, Writing -review \& editing.

\section{REFERENCES}

1. Sitarz R, Skierucha M, Mielko J, Offerhaus GJA, Maciejewski R, Polkowski WP. Gastric cancer: epidemiology, prevention, classification, and treatment. Cancer Manag Res. 2018;10:23948. https://doi.org/10.2147/CMAR.S149619

2. Charalampakis N, Economopoulou P, Kotsantis I, Tolia M, Schizas D, Liakakos T, et al. Medical management of gastric cancer: a 2017 update. Cancer Med 2018;7(1):123-33. https:// doi.org/10.1002/cam4.1274
3. Chen D, Zhou H, Liu G, Zhao Y, Cao G, Liu Q. SPOCK1 promotes the invasion and metastasis of gastric cancer through Sluginduced epithelial-mesenchymal transition. J Cell Mol Med. 2018;22(2):797-807. https://doi.org/10.1111/jcmm.13357

4. Okugawa $Y$, Toiyama $Y$, Inoue $Y$, Saigusa S, Kobayashi M, Tanaka K, et al. Late-onset peritoneal recurrence of advanced gastric cancer 20 years after primary resection. World I Surg Oncol. 2010;8:104. https://doi.org/10.1186/1477-7819-8-104 
5. Sawaki K, Kanda M, Miwa T, Umeda S, Tanaka H, Tanaka C, et al. Troponin 12 as a specific biomarker for prediction of peritoneal metastasis in gastric cancer. Ann Surg Oncol. 2018;25(7):208390. https://doi.org/10.1245/s10434-018-6480-z

6. Fu H, Yang $\mathrm{H}$, Xu Z, Wang B, Mao J, Li X, et al. Exosomal TRIM3 is a novel marker and therapy target for gastric cancer. J Exp Clin Cancer Res. 2018;37(1):162. https://doi.org/10.1186/ s13046-018-0825-0

7. Chen $\mathrm{TH}$, Lee $\mathrm{C}$, Chiu CT, Chu YY, Cheng HT, Hsu JT, et al. Circulating microRNA-196a is an early gastric cancer biomarker. Oncotarget. 2017;9(12):10317-23. https://doi.org/10.18632/ oncotarget.23126

8. Marabese M, Broggini M, Reijans M, Gouedard C, Maertens G, Sablon E, et al. Abstract 3739: Comparison of technologies for EGFR analysis within a subset of a randomized clinical trial [abstract]. In: Proceedings of the American Association for Cancer Research Annual Meeting 2017. Cancer Res. 2017;77(13 Suppl):Abstract nr 3739. https://doi.org/10.1158/1538-7445. AM2017-3739

9. Duan Z, He C, Gong $Y$, Li $P, X u$ Q, Sun LP, et al. Promoter polymorphisms in DNA repair gene ERCC5 and susceptibility to gastric cancer in Chinese. Gene. 2012;511(2):274-9. https:// doi.org/10.1016/j.gene.2012.09.025

10. Na N, Dun E, Ren L, Li G. Association between ERCC5 gene polymorphisms and breast cancer risk. Int J Clin Exp Pathol. 2015;8(3):3192. PMID: 26045839

11. Lu JJ, Zhang HQ, Mai P, Ma X, Chen X, Yang YX, et al. Lack of association between ERCC5 gene polymorphisms and gastric cancer risk in a Chinese population. Genet Mol Res. 2016;15(2). https://doi.org/10.4238/gmr.15027779

12. Namazi A, Forat-Yazdi $M$, Jafari MA, Foroughi $E$, Farahnak $S$, Nasiri R, et al. Association between polymorphisms of ERCC5 gene and susceptibility to gastric cancer: a systematic review and meta-analysis. Asian Pac J Cancer Prev. 2017;18(10):2611-7. https://doi.org/10.22034/APJCP.2017.18.10.2611

13. Yang LQ, Zhang $Y$, Sun HF. Investigation on ERCC5 genetic polymorphisms and the development of gastric cancer in a Chinese population. Genet Mol Res. 2016;15(3). https://doi. org/10.4238/gmr.15038364

14. Ruiz-Cosano J, Torres-Moreno D, Conesa-Zamora P. Influence of polymorphisms in ERCC5, XPA and MTR DNA repair and synthesis genes in B-cell lymphoma risk. A case-control study in Spanish population. J BUON. 2013;18(2):486-90. PMID: 23818366

15. Chen YZ, Guo F, Sun HW, Kong HR, Dai SJ, Huang SH, et al. Association between XPG polymorphisms and stomach cancer susceptibility in a Chinese population. J Cell Mol Med. 2016;20(5):903-8. https://doi.org/10.1111/jcmm.12773

16. Guo BW, Yang L, Zhao R, Hao SZ. Association between ERCC5 gene polymorphisms and gastric cancer risk. Genet Mol Res. 2016;15(2). https://doi.org/10.4238/gmr.15027828

17. Feng YB, Fan DQ, Yu J, Bie YK. Association between XPG gene polymorphisms and development of gastric cancer risk in a Chinese population. Genet Mol Res. 2016;15(2). https:// doi.org/10.4238/gmr.15027877

18. Chen JF, Liang HJ, Wang D, Tong JT, Liao LCC. Relationship between ERCC 5 promoter polymorphism and chemosensitivity of oxaliplatin-based chemotherapy in patients with advanced colorectal cancer. Chinese Clinical Oncology. 2009:105. Available from: http://en.cnki.com.cn/Article_en/CJFDTotalLCZL200910003.htm

19. Zhou H, Shi TY, Zhang W, Li Q, Zhu J, He J, et al. XPG gene rs751402 C>T polymorphism and cancer risk: evidence from 22 publications. Oncotarget. 2017;8(32):53613-22. https:// doi.org/10.18632/oncotarget.19421

20. Sun Y, Tan L, Li H, Qin X, Liu J. Association of NER pathway gene polymorphisms with susceptibility to laryngeal cancer in a Chinese population. Int J Clin Exp Pathol. 2015;8(9):1161521. PMID: 26617899

21. Yang WG, Zhang SF, Chen JW, Li L, Wang WP, Zhang XF. SNPs of excision repair cross complementing group 5 and gastric cancer risk in Chinese populations. Asian Pac $J$ Cancer Prev. 2012;13(12):6269-72. https://doi.org/10.7314/ apjcp.2012.13.12.6269

22. Zhang Z, Yin J, Xu Q, Shi J. Association between the XPG gene rs2094258 polymorphism and risk of gastric cancer. J Clin Lab Anal. 2018;32(8):e22564. https://doi.org/10.1002/ jcla.22564

23. Sang $L$, Lv Z, Sun LP, Xu Q, Yuan Y. Impact of SNP-SNP interactions of DNA repair gene ERCC 5 and metabolic gene GSTP1 on gastric cancer/atrophic gastritis risk in a Chinese population. World J Gastroenterol. 2018;24(5):602-12. https:// doi.org/10.3748/wjg.v24.i5.602

24. Zhang C, Liao Z, Yu G, Huang W, Song X. Study on association between ERCC5 single nucleotide polymorphism and susceptibility to esophageal cancer. J BUON. 2017;22(4):97984. PMID: 28952217

25. Zhu ML, Shi TY, Hu HC, He J, Wang M, Jin L, et al. Polymorphisms in the ERCC5 gene and risk of esophageal squamous cell carcinoma (ESCC) in Eastern Chinese populations. PLoS One. 2012;7(7):e41500. https://doi.org/10.1371/journal. pone. 0041500 